March 2, 2012 9:39 WSPC/130-JCA ws-jca

Journal of Computational Acoustics

(C) IMACS

\title{
A LOW-HIGH FREQUENCY FORMULATION FOR FAST ACOUSTIC CALCULATIONS
}

\author{
SÉBASTIEN BESSET \\ Laboratoire de Tribologie et Dynamique des Systèmes \\ École Centrale de Lyon, 69134 Écully cedex, France \\ sebastien.besset@ec-lyon.fr \\ Received (Day Month Year) \\ Revised (Day Month Year)
}

\begin{abstract}
Noise predictions in acoustic cavities lead to different formulations in low or high frequency ranges.
In the case of fast calculations, we propose a mixed formulation based on a finite element mesh including a "high frequency energy degree of freedom" to predict high frequency phenomena. The mesh size is planned for studying low-(medium) frequency range phenomena through a classical finite element method. The same mesh is used to build energy elements to take into account the (medium)-high frequency range phenomena. The method aims at performing low-cost calculations in medium-high frequency ranges using low-frequency based existing meshes, i.e. without re-meshing the system.
\end{abstract}

Keywords: Energy methods; Large frequency range problems. 


\section{Introduction}

The problem of predicting the noise in the acoustic cavities has been discussed by many authors. The methods are generally quite different for low and high frequency issues. Low frequency phenomena are most often treated through classical finite element methods or boundary element methods.

In medium-high frequency ranges, energy methods are often proposed, since finite element methods would require finer mesh that would lead to high calculation costs. Moreover, the modal overlap in the medium-high frequency domain legitimizes the use of averaged energy variables. Among the most commonly used methods, let's mention the Statistical Energy Analysis (SEA) ${ }^{6}$. The SEA was extended by Nefske and Sung ${ }^{7}$ who proposed a local energy formulation, whereas the SEA is based on global energies of subsystems. The method proposed in this paper is based on the Simplified Energy Method (called MES in the french terminology) we already described in former papers ${ }^{14,15}$.

The Simplified Energy Method (MES) comes from the local energy formalism introduced by Nefske and Sung ${ }^{7}$ and improved by many authors ${ }^{8,3,4,9}$. In the context of acoustic noise prediction, it was applied in several papers ${ }^{10,11,12}$. The lack of precision in medium frequency range was already addressed by Besset, Ichchou and Jézéquel ${ }^{14}$ who proposed a mixed BEM/MES formulation. The aim of the method was to describe the direct field through a classical BEM method, whereas the reverberated field was described by the MES method. Nevertheless, this method needs a fine modelization of the structure around the injected sources, which is often costly. 
In this paper, we address the problem of the fast prediction of acoustic fields in a large frequency band. We propose a computational method to estimate the noise level in a cavity in low, medium and high frequency ranges using a classical finite element mesh. In the low frequency domain, a classical finite element method is used, which is valid until a certain frequency $f_{c}$. In order to reduce the size of the finite element problem, we apply component mode synthesis methods developed in former papers ${ }^{2,5}$ and called triple modal synthesis method. For frequencies higher than $f_{c}$, we seek to adapt a MES formulation to the existing finite element mesh. Hence, the resulting degrees of freedom will be a mix between generalized degrees of freedom coming from the modal synthesis and energy degrees of freedom.

\section{Finite element formulation}

\subsection{Low frequency modelisation}

The considered system (see Figure 1) is made of a simple structure made of plates coupled to an acoustic cavity.

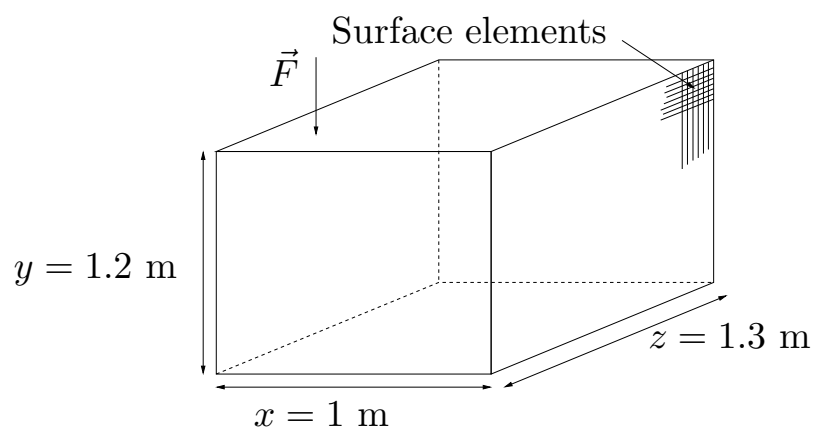

Fig. 1. Structure to be studied 
In the low frequency domain, we consider displacement degrees of freedom $\boldsymbol{X}=$ $\{\boldsymbol{u} \boldsymbol{v} \boldsymbol{w}\}^{T}$ for the plates and pressure degrees of freedom $\boldsymbol{p}$ for the fluid, hence the following motion equation:

$$
\left(\left[\begin{array}{cc}
\boldsymbol{K}_{x x} & \boldsymbol{K}_{x p} \\
\mathbf{0} & \boldsymbol{K}_{p p}
\end{array}\right]-\omega^{2}\left[\begin{array}{cc}
\boldsymbol{M}_{x x} & \mathbf{0} \\
\boldsymbol{M}_{p x} & \boldsymbol{M}_{p p}
\end{array}\right]\right)\left\{\begin{array}{l}
\boldsymbol{X} \\
\boldsymbol{p}
\end{array}\right\}=\left\{\begin{array}{l}
\boldsymbol{F} \\
\mathbf{0}
\end{array}\right\}
$$

Equation 1 can be found in ${ }^{1}$. The structure mesh is a $2 \mathrm{D}$ mesh, it can then be quite fine. In this paper we consider quadrilateral -4 node $20 \mathrm{DOF}$ - plate elements. The 3D mesh concerning the acoustic cavity cannot be so fine to avoid high calculation costs. Hence, the dynamic behaviour of the structure can be predicted for higher frequency bands, whereas the behaviour of the acoustical part of the system cannot. It is the reason why we add energy variables in the acoustic domain. In this paper we consider 8-node 8-DOF hexahedron elements. In the low frequency domain, the displacement and pressure fields can be expressed as functions of the modes of the structure. First, the degrees of freedom of the plates are split into surface degrees of freedom $\boldsymbol{X}_{s}=\left\{\boldsymbol{u}_{s} \boldsymbol{v}_{s} \boldsymbol{w}_{s}\right\}^{T}$ and boundary degrees of freedom $\boldsymbol{X}_{b}=\left\{\boldsymbol{u}_{b} \boldsymbol{v}_{b} \boldsymbol{w}_{b}\right\}^{T}$, which leads to the following mass and stiffness matrices:

$$
\boldsymbol{K}_{x x}=\left[\begin{array}{cc}
\boldsymbol{K}_{x_{s} x_{s}} & \boldsymbol{K}_{x_{s} x_{b}} \\
\boldsymbol{K}_{x_{b} x_{s}} & \boldsymbol{K}_{x_{b} x_{b}}
\end{array}\right], \quad \boldsymbol{M}_{x x}=\left[\begin{array}{c}
\boldsymbol{M}_{x_{s} x_{s}} \boldsymbol{M}_{x_{s} x_{b}} \\
\boldsymbol{M}_{x_{b} x_{s}} \boldsymbol{M}_{x_{b} x_{b}}
\end{array}\right], \quad \boldsymbol{F}=\left\{\begin{array}{c}
F_{s} \\
F_{b}
\end{array}\right\}
$$

The acoustic degrees of freedom $p$ are then described using cavity modes $\Phi_{p}$, the degrees of freedom concerning the plates are described using fixed modes $\Phi_{s}$ and constraint modes $\Psi_{s}$, and the degrees of freedom of the boundaries are described using "branch modes" $\Phi_{b}$ : 


$$
\left\{\begin{array}{l}
\boldsymbol{p}=\Phi_{p} \boldsymbol{q}_{p} \\
\left\{\begin{array}{lll}
\boldsymbol{u}_{s} & \boldsymbol{v}_{s} & \left.\boldsymbol{w}_{s}\right\}^{T}=\Phi_{s} \boldsymbol{q}_{s}+\Psi_{s}\left\{\boldsymbol{u}_{b} \boldsymbol{v}_{b} \boldsymbol{w}_{b}\right\}^{T} \\
\left\{\boldsymbol{u}_{b}\right. & \boldsymbol{v}_{b} & \boldsymbol{w}_{b}
\end{array}\right\}^{T}=\Phi_{b} \boldsymbol{q}_{b}
\end{array}\right.
$$

$\Psi_{s}, \Phi_{p}, \Phi_{s}$ and $\Phi_{b}$ are obtained by solving the following equations:

$$
\left\{\begin{array}{l}
\left(\boldsymbol{K}_{p p}-\omega^{2} \boldsymbol{M}_{p p}\right) \Phi_{p}=\mathbf{0} \\
\left(\boldsymbol{K}_{x_{s} x_{s}}-\omega^{2} \boldsymbol{M}_{x_{s} x_{s}}\right) \Phi_{s}=\mathbf{0} \\
\Psi_{s}=-\boldsymbol{K}_{x_{s} x_{s}}^{-1} \boldsymbol{K}_{x_{s} x_{p}} \\
{\left[\left(\left\{\Psi_{s} \Psi_{s}\right\} \boldsymbol{K}_{x x}\left\{\Psi_{s} \Psi_{s}\right\}^{T} \boldsymbol{K}_{x x}\right)-\omega^{2}\left(\left\{\Psi_{s} \Psi_{s}\right\} \boldsymbol{M}_{x x}\left\{\Psi_{s} \Psi_{s}\right\}^{T}\right)\right] \Phi_{b}=\mathbf{0}}
\end{array}\right.
$$

Equation 1 becomes then:

$$
\left(\left[\begin{array}{cc|c}
\tilde{\boldsymbol{k}}_{x_{b} x_{b}} & \tilde{\boldsymbol{K}}_{x_{b} x_{s}} & \\
\tilde{\boldsymbol{K}}_{x_{b} x_{s}} & \tilde{\boldsymbol{k}}_{x_{s} x_{s}} & \\
\hline \mathbf{0} & \tilde{\boldsymbol{K}}_{p p}
\end{array}\right]-\omega^{2}\left[\begin{array}{cc|c}
\tilde{\boldsymbol{m}}_{x_{b} x_{b}} & \mathbf{0} & \mathbf{0} \\
\mathbf{0} & \tilde{\boldsymbol{m}}_{x_{s} x_{s}} & \\
\hline \tilde{\boldsymbol{M}}_{p x} & \tilde{\boldsymbol{m}}_{p p}
\end{array}\right]\right)\left\{\begin{array}{c}
\boldsymbol{q}_{b} \\
\boldsymbol{q}_{s} \\
\boldsymbol{q}_{p}
\end{array}\right\}=\left\{\begin{array}{c}
\tilde{\boldsymbol{F}}_{b} \\
\tilde{\boldsymbol{F}}_{s} \\
\mathbf{0}
\end{array}\right\}
$$

where lower case matrices $\tilde{\boldsymbol{k}}_{i j}$ and $\tilde{\boldsymbol{m}}_{i j}$ are diagonal matrices.

\subsection{Energy formulation - low frequency domain}

As we are interested in the noise inside the cavity, we will consider the energy variable $W=\frac{|p|^{2}}{\left|p_{\text {ref }}\right|^{2}}$. In the low frequency domain, $W$ is obtained from equation 3 :

$$
W_{\mathrm{LF}}^{n}=\frac{1}{\left|p_{\mathrm{ref}}\right|^{2}}\left|\Phi_{p}^{n} \boldsymbol{q}_{p}\right|^{2}
$$


where $N_{p}$ is the number of cavity modes and $\Phi_{p}^{n}$ is the $n^{\text {th }}$ line of matrix $\Phi_{p}$. Moreover, equation 5 leads to the following expression of $q_{p}$ :

$$
\boldsymbol{q}_{p}=\mathcal{G}(\omega)\left\{\tilde{\boldsymbol{F}}_{b} \tilde{\boldsymbol{F}}_{s} \mathbf{0}\right\}^{T}
$$

where $\mathcal{G}(\omega)$ is a matrix obtained by inverting the left side of equation 5 . Hence the following derivation:

$$
\begin{aligned}
W_{\mathrm{LF}}^{n} & =\frac{1}{\left|p_{\mathrm{ref}}\right|^{2}}\left|\Phi_{p}^{n} \mathcal{G}(\omega)\left\{\tilde{\boldsymbol{F}}_{b} \tilde{\boldsymbol{F}}_{s} \mathbf{0}\right\}^{T}\right|^{2} \\
& =\frac{1}{\left|p_{\mathrm{ref}}\right|^{2}}\left(\Phi_{p}^{n} \mathcal{G}(\omega)\left\{\tilde{\boldsymbol{F}}_{b} \tilde{\boldsymbol{F}}_{s} \mathbf{0}\right\}^{T}\right)\left(\Phi_{p}^{n} \mathcal{G}(\omega)\left\{\tilde{\boldsymbol{F}}_{b} \tilde{\boldsymbol{F}}_{s} \mathbf{0}\right\}^{T}\right)^{T *} \\
& =\frac{1}{\left|p_{\mathrm{ref}}\right|^{2}}(\Phi_{p}^{n} \mathcal{G}(\omega) \underbrace{\left\{\tilde{\boldsymbol{F}}_{b} \tilde{\boldsymbol{F}}_{s} \mathbf{0}\right\}^{T}\left\{\tilde{\boldsymbol{F}}_{b}^{*} \tilde{\boldsymbol{F}}_{s}^{*} \mathbf{0}\right\}}_{\mathbf{F}} \mathcal{G}^{T *}(\omega) \Phi_{p}^{n T *}) \\
& =\frac{1}{\left|p_{\mathrm{ref}}\right|^{2}}(\underbrace{\Phi_{p}^{n} \mathcal{G}(\omega)}_{\mathcal{I}(\omega)} \mathbf{F} \underbrace{\mathcal{G}^{T *}(\omega) \Phi_{p}^{n T *}}_{\mathcal{J}(\omega)}) \\
& =\frac{1}{\left|p_{\mathrm{ref}}\right|^{2}}(\mathcal{I}(\omega) \mathbf{F} \mathcal{J}(\omega))
\end{aligned}
$$

where $(a+i b)^{*}=a-i b$ and $\boldsymbol{X}^{T}$ is the transpose of matrix $\boldsymbol{X}$.

\subsection{Energy formulation - Medium-high frequency domain}

In this section, we explain how to compute the energy variable $W$ for the medium-high frequency domain using the finite element mesh already used for the classical finite element formulation.

The energy density inside the cavity can be expressed as a function of the kinematic 
degrees of freedom on the boundaries:

$$
\boldsymbol{W}=\mathcal{H}\left(\left\{\begin{array}{lll}
\boldsymbol{X}_{b} & \boldsymbol{X}_{s} & \boldsymbol{p}
\end{array}\right\}^{T}\right)
$$

The aim of this section is to explain how to obtain $\mathcal{H}$. Let note $N_{2}$ the number of elements of the structure (i.e. the surface elements shown in Figure 1) and $N_{3}$ the number of nodes of the 3D mesh (i.e. the acoustic mesh). The dimension of vector $\boldsymbol{W}$ will be $\left(N_{3}, 1\right)$ and it can be expressed as follows:

$$
W_{n}=\underbrace{\sum_{m=1}^{N_{2}} \frac{\Re\left(i \omega p_{m} X_{m}^{\perp^{*}}\right)}{2 c_{0}} \iint_{S_{m}} \frac{\cos \theta}{\pi r_{n m}^{2}} \mathrm{dP}}_{\text {Direct field }}+\underbrace{\sum_{m=1}^{N_{2}} \frac{\sigma_{m}}{c_{0}} \iint_{S_{m}} \frac{\cos \theta}{\pi r_{n m}^{2}} \mathrm{dP}}_{\text {Reverberated field }} \quad \text { for } n=\left[1, \ldots, N_{3}\right]
$$

where:

- $S_{m}$ is the surface of element $m$;

- $r_{n m}$ is the distance between point $n$ and the center of element $m$;

- $c_{0}$ is the sound velocity in the cavity;

- $X_{m}^{\perp *}$ in the component of the displacement orthogonal to element $m$ (i.e. colinear to vector $\vec{n}$ given in Figure 2);

- $\theta$ is an angle defined in figure 2. $\frac{\cos \theta}{\pi}$ corresponds to the directivity on the boundary. It is assumed to be lambertian but another value may be proposed;

- $\sigma_{m}$ is the reverberated energy density on the boundaries.

In order to obtain $\boldsymbol{W}$ as a function of the injected power $\mathcal{P}_{m}=\frac{1}{2} \Re\left(i \omega p_{m} X_{m}^{\perp^{*}}\right)$ on the 
March 2, 2012 9:39 WSPC/130-JCA ws-jca

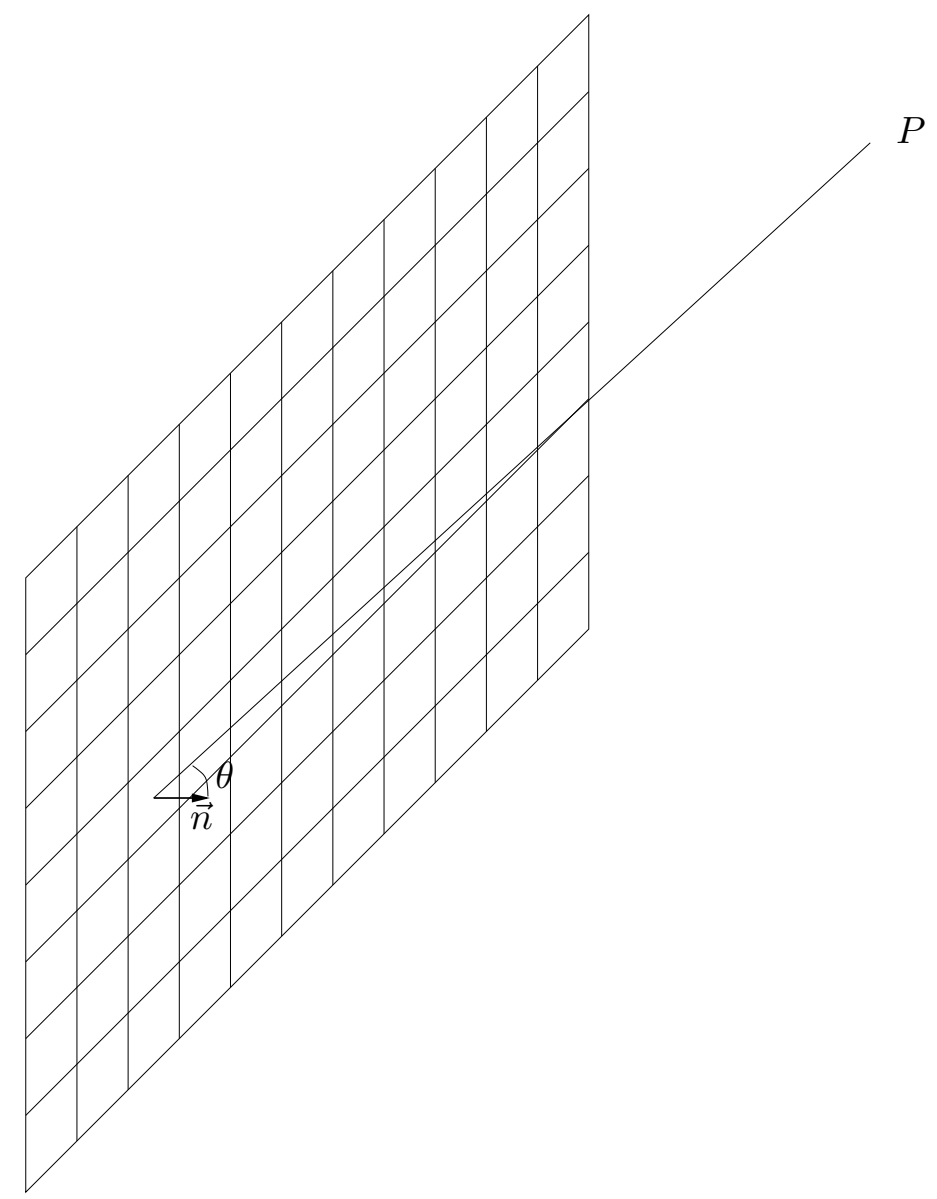

Fig. 2. Use of the FEM mesh to compute energy variables

boundaries, we need to express $\sigma_{m}$ as a function of $\mathcal{P}_{m}$. Let's consider the power balance on one element of the structure. Considerint two elements $\xi$ and $\zeta$, the power balance can be written as follows:

$$
\sigma_{\xi}=\frac{1-\alpha_{\xi}}{S_{\xi}} \sum_{\substack{\zeta=1 \\ \zeta \neq \xi}}\left[\left(\mathcal{P}_{\zeta}+\sigma_{\zeta}\right) \iint_{S_{\xi}} \iint_{S_{\zeta}} \frac{\cos \theta_{\xi} \cos \theta_{\zeta}}{\pi r_{\xi \zeta}^{4}} \mathrm{dP}_{\xi} \mathrm{dP}_{\zeta}\right]
$$

where $r_{\xi \zeta}=P_{\xi} P_{\zeta}$ (distance between $P_{\xi}$ and $P_{\zeta}$ ). $\alpha_{\xi}$ is the absorption coefficient of 
element $\xi . S_{\xi}$ is the area of element $\xi$. Equation 11 can also be written with a matrix notation as follows, introducing a square matrix $\boldsymbol{Q}$ :

$$
\left\{\begin{array}{llll}
\sigma_{1} & \ldots & \sigma_{N_{2}}
\end{array}\right\}^{T}=\boldsymbol{Q}\left\{\mathcal{P}_{1} \ldots \mathcal{P}_{N_{2}}\right\}^{T}
$$

Matrix $\boldsymbol{Q}$ is obtained by writing equation 11 for $\xi=1,2, \ldots, N_{2}$ with $\sigma_{i}$ in the left terms and $P_{i}$ in the right terms, which leads to a matrix equation including matrix $\boldsymbol{Q}$. Hence the following matrix equation allowing to obtain $\boldsymbol{W}_{\mathrm{HF}}$ the energy density for medium-high frequency domains:

$$
\boldsymbol{W}_{\mathrm{HF}}=\left\{\begin{array}{lll}
W_{1} & \ldots & W_{N_{2}}
\end{array}\right\}^{T}=\boldsymbol{R}\left\{\mathcal{P}_{1} \ldots \mathcal{P}_{N_{2}}\right\}^{T}
$$

Matrix $\boldsymbol{R}$ is obtained by including equation 12 in equation 10 . Hence, the $\mathrm{n}^{\text {th }}$ line of vector $\boldsymbol{W}_{\mathrm{HF}}$ can be written as follows:

$$
\begin{aligned}
\boldsymbol{W}_{\mathrm{HF}}^{n} & =\boldsymbol{R}^{n}\left\{\mathcal{P}_{1} \ldots \mathcal{P}_{N_{2}}\right\}^{T} \\
& =\boldsymbol{R}^{n} \frac{1}{2} \Re\left(\left\{p_{1} v_{1}^{*} \ldots p_{N_{2}} v_{N_{2}}^{*}\right\}^{T}\right) \\
& =\boldsymbol{R}^{n} \frac{\omega}{2} \Re\left(\left\{p_{1} X_{1}^{*} \ldots p_{N_{2}} X_{N_{2}}^{*}\right\}^{T}\right)
\end{aligned}
$$

where $\boldsymbol{R}^{n}$ is the $n^{\text {th }}$ line of matrix $\boldsymbol{R}$. Let's now express $p_{i}$ and $X_{i}$ as functions of $\left\{\tilde{\boldsymbol{F}}_{b} \tilde{\boldsymbol{F}}_{s} \mathbf{0}\right\}^{T}:$ 


$$
\begin{aligned}
& p_{i}=\Phi_{p}^{i} \boldsymbol{q}_{p} \\
& =\underbrace{\Phi_{p}^{i} \mathcal{G}(\omega)}_{\mathcal{K}_{i}(\omega)}\left\{\tilde{\boldsymbol{F}}_{b} \tilde{\boldsymbol{F}}_{s} \mathbf{0}\right\}^{T} \\
& X_{i}=\left\{\begin{array}{ll}
X_{s} & X_{b}
\end{array}\right\}^{T} \\
& =\left[\begin{array}{cc}
\Phi_{s} & \Psi_{s} \Phi_{b} \\
0 & \Phi_{b}
\end{array}\right]\left\{\begin{array}{l}
q_{s} \\
q_{b}
\end{array}\right\} \\
& =\underbrace{\left[\begin{array}{cc}
\Phi_{s} & \Psi_{s} \Phi_{b} \\
0 & \Phi_{b}
\end{array}\right]\left\{\begin{array}{l}
\alpha(\omega) \\
\beta(\omega)
\end{array}\right\}}_{\mathcal{L}_{i}(\omega)}\left\{\tilde{\boldsymbol{F}}_{b} \tilde{\boldsymbol{F}}_{s} \mathbf{0}\right\}^{T}
\end{aligned}
$$

where $\alpha(\omega)$ and $\beta(\omega)$ are matrices obtained from equation 5. Hence the following expression for $p_{i} X_{i}^{*}$ :

$$
p_{i} X_{i}^{*}=\mathcal{K}_{i}(\omega) \underbrace{\left\{\tilde{\boldsymbol{F}}_{b} \tilde{\boldsymbol{F}}_{s} \mathbf{0}\right\}^{T}\left\{\tilde{\boldsymbol{F}}_{b} \tilde{\boldsymbol{F}}_{s} \mathbf{0}\right\}^{*}}_{\mathbf{F}} \mathcal{L}_{i}^{*}(\omega)
$$

Equation 14 leads then to the following expression:

$$
\begin{aligned}
W_{\mathrm{HF}}^{n} & =\frac{1}{2} \omega \boldsymbol{R}^{n} \operatorname{Diag}\left(\left[\begin{array}{c}
\mathcal{K}_{1}(\omega) \\
\vdots \\
\mathcal{K}_{N_{2}}(\omega)
\end{array}\right] \mathbf{F}\left[\mathcal{L}_{1}^{*}(\omega) \ldots \mathcal{L}_{N_{2}}^{*}\right]\right) \\
= & \frac{1}{2} \omega \boldsymbol{R}^{n} \operatorname{Diag}\left(\mathcal{K}(\omega) \mathbf{F} \mathcal{L}^{T}(\omega)\right)
\end{aligned}
$$




\subsection{Energy formulation - Large frequency domain}

The aim of this section is to express $W$ in a large frequency domain using the formulations given in the last sections. According to the expressions found for $W_{\mathrm{LF}}$ and $W_{\mathrm{HF}}$ we define the quantity $\left\{W_{\mathrm{LF}}^{n} W_{\mathrm{HF}}^{n}\right\}^{T}$ as follows:

$$
\left\{\begin{array}{c}
W_{\mathrm{LF}}^{n} \\
W_{\mathrm{HF}}^{n}
\end{array}\right\}=\left\{\begin{array}{c}
\frac{1}{2} \omega \boldsymbol{R}^{n} \operatorname{Diag}\left(\mathcal{K}(\omega) \mathbf{F} \mathcal{L}^{T}(\omega)\right) \\
\mathcal{I}(\omega) \mathbf{F} \mathcal{J}(\omega)
\end{array}\right\}
$$

Let's introduce two filter functions $\mathcal{F}_{\mathrm{LF}}\left(f_{c}\right)$ and $\mathcal{F}_{\mathrm{HF}}\left(f_{c}\right)$ depending on a given frequency $f_{c}$. If $\omega<2 \pi f_{c}, W_{L F}$ should mainly be considered, whereas if $w>2 \pi f_{c}, W_{H F}$ should mainly be considered. Hence the global formulation:

$$
W=\mathcal{F}_{\mathrm{LF}}\left(f_{c}\right) W_{L F}+\mathcal{F}_{\mathrm{HF}}\left(f_{c}\right) W_{H F}
$$

\section{Results}

The system considered for the numerical example is given in figure 1. It is an acoustic cavity bounded by 6 plates. The figure 3 shows the results found for $W, W_{\mathrm{LF}}, W_{H F}$ and $W_{\text {ref }}$ :

- $W, W_{\mathrm{LF}}$ and $W_{H F}$ are computed using a fine 2D mesh and a large 3D mesh;

- $W_{\text {ref }}$ is computed using a very fine mesh and is considered as the reference. It is the result of a direct finite element calculation. The mesh size has been chosen in order to obtain accurate results. 
Table 1 shows that the matrices needed for computing the reference solution is 100 times larger than the matrices needed for compution the solution with the other methods. As a consequence the numerical operations count is 100 times larger if a standard FEM approach is used. Of course, given a fixed tolerance we can only argue aboutthe relative computational cost of the different methods.

\begin{tabular}{r|ccc} 
& Size of matrices & LF & HF \\
\hline Reference (FEM) & $569 \times 569$ & $\bullet$ & $\bullet$ \\
FEM (LF) & $61 \times 61$ & $\bullet$ & - \\
Energy formulation (HF) & $61 \times 61$ & - & $\bullet$ \\
Proposed method & $61 \times 61$ & $\bullet$ & $\bullet$
\end{tabular}

Table 1. Size of matrices

In this example, functions $\mathcal{F}_{\mathrm{LF}}\left(f_{c}\right)$ and $\mathcal{F}_{\mathrm{HF}}\left(f_{c}\right)$ are defined as follows:

$$
\begin{aligned}
& \mathcal{F}_{\mathrm{LF}}\left(f_{c}\right)=\frac{1}{\pi}\left(\tan ^{-1}\left[n_{c}\left(\omega-2 \pi f_{c}\right)\right]+\frac{\pi}{2}\right) \\
& \mathcal{F}_{\mathrm{HF}}\left(f_{c}\right)=\frac{1}{\pi}\left(\tan ^{-1}\left[n_{c}\left(-\omega-2 \pi f_{c}\right)\right]+\frac{\pi}{2}\right)
\end{aligned}
$$

where $n_{c}$ is an integer (we choose here $n_{c}=6$ ). If the frequency $f=\frac{\omega}{2 \pi}$ is greater than $f_{c}$, we consider that the low frequency formulation is no more valuable. We choose $f_{c}=290 \mathrm{~Hz}$ for this example. It must be noticed that the choice of the filter functions is arbitrary. In fact, it is difficult to choose such functions: the frequency $f_{c}$ must be carefully set and $\mathcal{F}_{\mathrm{LF}}\left(f_{c}\right)$ and $\mathcal{F}_{\mathrm{HF}}\left(f_{c}\right)$ must satisfy the following equations: 
March 2, 2012 9:39 WSPC/130-JCA ws-jca

$$
\left\{\begin{array}{l}
\lim _{\omega \rightarrow 0}\left[\mathcal{F}_{\mathrm{LF}}\left(f_{c}\right)\right]=1 \\
\lim _{\omega \rightarrow+\infty}\left[\mathcal{F}_{\mathrm{LF}}\left(f_{c}\right)\right]=0 \\
\lim _{\omega \rightarrow 0}\left[\mathcal{F}_{\mathrm{HF}}\left(f_{c}\right)\right]=0 \\
\lim _{\omega \rightarrow+\infty}\left[\mathcal{F}_{\mathrm{HF}}\left(f_{c}\right)\right]=1
\end{array}\right.
$$

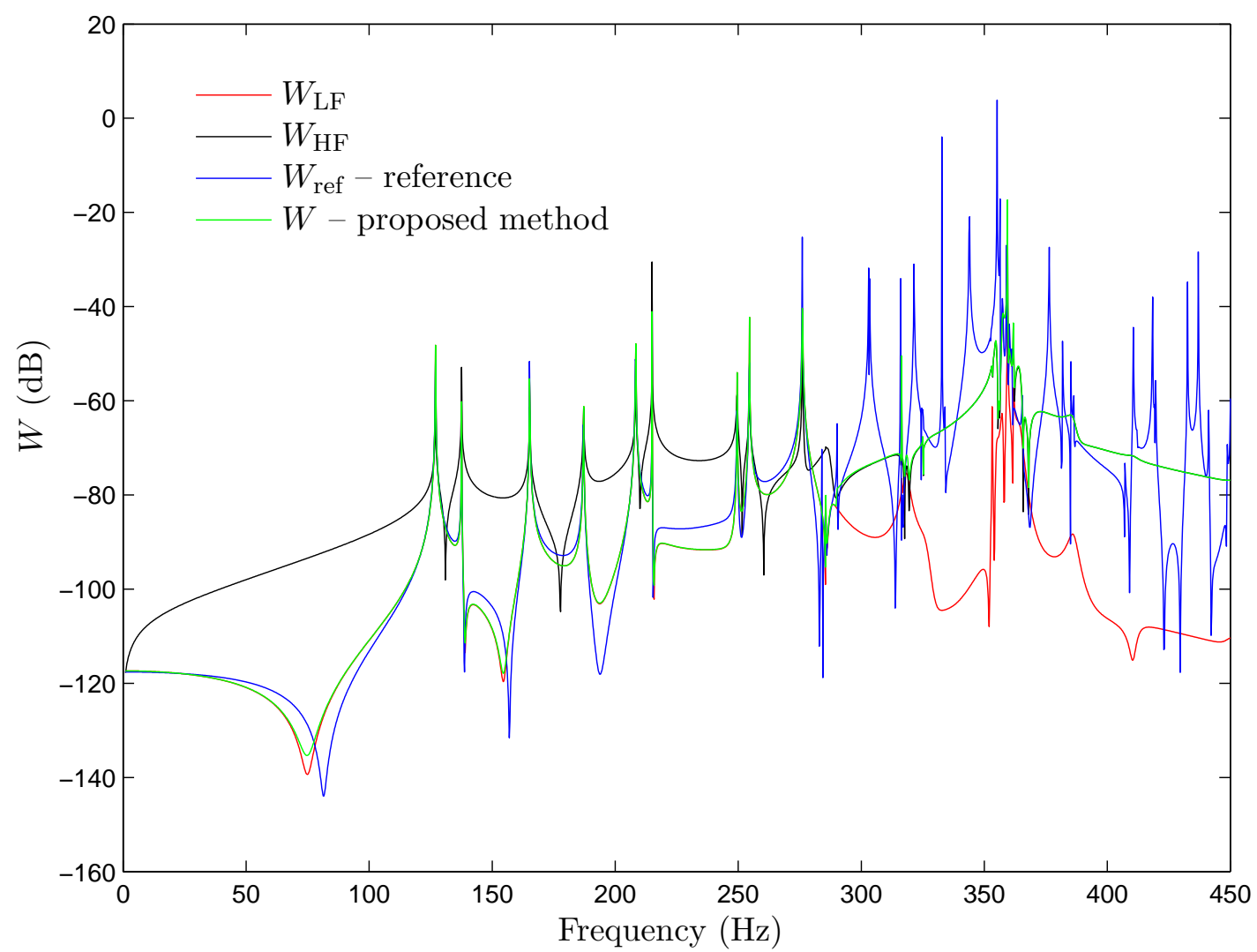

Fig. 3. Power density in the cavity

The results show that the proposed method allows to obtain good results in a large frequency band: For the low frequency range, the proposed method is close to the reference, 
all the peaks are at the right place. For the medium-high frequency range, the averaged response of the system is correct and very close to the energy method $\left(W_{H F}\right)$. It is interesting to note that the average power density for the higher frequencies shows some peaks. This is due to the fact that the injected power is provided by the finite element formulation of the boundaries and contains modal information.

\section{Conclusion}

In this paper we propose to use a classical finite element mesh to estimate the mediumhigh frequency behaviour of an acoustic cavity. The aim is to get a good estimation without increasing too much the calculation cost, i.e. using the already meshed structure. The results presented in the paper show that the method allow to predict the large frequency range behaviour of the structure considering only few degrees of freedom. Nevertheless, several points remain to study. First, the choice of $f_{c}$ and functions $\mathcal{F}_{\mathrm{LF}}$ and $\mathcal{F}_{\mathrm{HF}}$ is not easy. Moreover, the medium-high frequency estimation needs the pressure on the boundaries of the system. However, this pressure field is obtained by a classic finite element method, which is not valuable in this frequency domain. We are currently working on the improvement of the method considering this last point.

\section{List of variables}

\subsection{Finite element formulation}

- $\boldsymbol{X}=\left\{\boldsymbol{X}_{s}, \boldsymbol{X}_{b}\right\}^{T}$ : structural degrees of freedom; 
- $\boldsymbol{X}_{s}$ : surface degrees of freedom;

- $\boldsymbol{X}_{b}$ : boundary degrees of freedom;

- $\boldsymbol{p}$ : pressure degrees of freedom;

- $\boldsymbol{K}, \boldsymbol{M}$ : stiffness and mass matrices;

- $\Phi_{p}$ : acoustic modes;

- $\Phi_{s}$ : fixed interface modes;

- $\Psi_{s}$ : constraint modes;

- $\Phi_{b}$ : "branch modes";

- $\tilde{\boldsymbol{K}}, \tilde{\boldsymbol{M}}$ : reduced stiffness and mass matrices;

- $\left\{\boldsymbol{q}_{b} \boldsymbol{q}_{s} \boldsymbol{q}_{p}\right\}^{T}$ : generalized degrees of freedom;

\subsection{Energy formulation}

- $S_{m}$ : surface of element $m$;

- $r_{n m}$ : distance between point $n$ and the center of element $m$;

- $c_{0}$ : sound velocity in the cavity;

- $X_{m}^{\perp^{*}}$ : component of the displacement orthogonal to element $m$ (i.e. colinear to vector $\vec{n}$ given in Figure 2);

- $\theta$ : angle defined in figure 2. $\frac{\cos \theta}{\pi}$ corresponds to the directivity on the boundary. It is assumed to be lambertian but another value may be proposed;

- $\sigma_{m}$ : reverberated energy density on the boundaries;

- $\Re(x)$ : real part of $x$;

- $\mathcal{P}_{m}$ : injected power. 


\section{References}

1. H. J.-P. Morand and R. Ohayon, Fluid structure interaction: Applied numerical methods, Wiley (Chichester and New York and Paris), 1995

2. S. Besset and L. Jézéquel, Dynamic Substructuring based on a Double Modal Analysis, J. Vib. Acoust, 130 (1), 2008

3. O. M. Bouthier, R. J. Bernhard, Simple models of energy flow in vibrating membranes, Journal of Sound and Vibration 182 (1) (1995) 129-147.

4. O. M. Bouthier, R. J. Bernhard, Simple models of the energetics of transversely vibrating plates, Journal of Sound and Vibration 182 (1) (1995) 149-164.

5. S. Besset and L. Jézéquel, Vibroacoustical Analysis Based on a Multimodal Strategy: Triple Modal Synthesis, J. Vib. Acoust, 130 (3), 2008

6. R. H. Lyon, Statistical Energy Analysis of Dynamical Systems: Theory and Application, Cambridge, Massachusetts, MIT Press, 1975.

7. D. Nefske, S. Sung, Power flow finite element analysis of dynamic systems: Basic theory and application to beams, NCA Publication 3.

8. J. Wohlever, R. Bernhard, Mechanical energy flow models of rods and beams, Journal of Sound and Vibration 153 (1992) 1-19.

9. Y. Lase, M. Ichchou, L. Jezequel, Energy analysis of bars an beams: Theoretical formulations, Journal of Sound and Vibration 192 (1996) 281-305.

10. A. Wang, N. Vlahopoulos, K. Wu, Development of an energy boundary element formulation for computing high-frequency sound radiation from incoherent intensity boundary conditions, Journal of Sound and Vibration 278 (2004) 413-436.

11. V. Cotoni, Modélisation de phénomènes vibroacoustiques en moyenne et hautes fréquences par méthode énergétique locale, Ph.D. thesis, Ecole Centrale de Lyon (2001). 
March 2, 2012 9:39 WSPC/130-JCA ws-jca

12. V. Cotoni, A. Le Bot, L. Jezequel, High frequency radiation of l-shaped plates by a local energy flow approach, Journal of Sound and Vibration 250 (3) (2002) 431-444.

13. L. Brillouin, Wave propagation in periodic structures, Dover Publications,Inc.,New York, 1953.

14. S. Besset, M.N. Ichchou and L. Jzquel, A coupled BEM and energy flow method for mid-high frequency internal acoustic, Journal of Computational Acoustic, 18(1) (2010) 69-85

15. M. Chabchoub, S. Besset and M.N. Ichchou, Identification of acoustic sources through an inverse energy method, Inverse Problems in Science \& Engineering 19(7) (2011) 903-919. 